\title{
Article
}

\section{Membrane Potential: Any diffusion?}

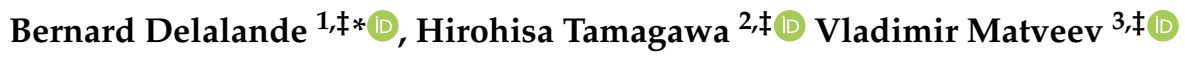 \\ 1 280, avenue de la Pierre Dourdant, 38290 La Verpilliere, France; bernard@somasimple.com \\ 2 Department of Mechanical Engineering, Faculty of Engineering, Gifu University, 1-1 Yanagido, Gifu, Gifu, \\ 501-1193 Japan; tmgwhrhs@gifu-u.ac.jp \\ 3 Laboratory of Cell Physiology, Institute of Cytology, Russian Academy of Sciences, Tikhoretsky Ave 4, St. \\ Petersburg 194064, Russia; vladimir.matveev@gmail.com \\ * Correspondence: bernard@somasimple.com \\ $\ddagger \quad$ These authors contributed equally to this work.
}

\begin{abstract}
The generation and maintenance of membrane potential is a fundamental part of Membrane Pump Theory. One of the key points of this hypothesis is based on a natural or facilitated molecular diffusion through several types of ion channels and pumps like the $\mathrm{Na} / \mathrm{K}$ ATPase. Chemistry, physics and especially electrochemistry, however, bring strong contradictions to this theoretical assumption. By respecting the principles of chemistry and electrostatics, it becomes obvious that this theoretical hypothesis cannot work. The ionic diffusion that would be at the origin of this potential cannot take place. Indeed, the topology and the forces involved definitively exclude the current model, which must absolutely be revised according to the current state of our knowledge and allow an advance in the understanding of the phenomena and open new research perspectives.
\end{abstract}

Keywords: membrane pump theory; membrane potential; diffusion; Biophysics; Biology

Introduction This article is the first part of a series on membrane theory and its contradictions.

- Any diffusion?

- The enigma of ion pumps

- The ignored shape

- The smoothing of math

The basic theoretical principle of the membrane potential generation seems to be very simple and has been modified many times to try to obtain more accuracy in its calculation. The original assumption is as follows:

The membrane would be semi-permeable to the various ions present in the external and intra-cellular environment. The cell also contains negative valence molecules, peptides and proteins, which are considered to be poorly mobile but unable to cross the membrane.

Potassium is more abundant in the cell than the cell outside and should therefore play a central role in generating this membrane potential [1]. As potassium is more abundant in the cell, there is a concentration difference between inside and outside the cell.

This concentration gradient is thought to induce an efflux of potassium out of the cell because potassium tends to move from the more concentrated area to the less concentrated area. When potassium leaves the cell, the electric field generated across the plasma membrane changes with the amount of potassium ions leaving the cell.

A sort of steady state is reached when the outflow of potassium caused by the simple diffusion is counterbalanced by the electrostatic force exerted to the potassium which tens to flow outward. What is so far described is true for the rest of mobile ions as well. Membrane potential (MP) is realized when such a steady state is achieved [2].

Membrane theory states that the generation of the membrane potential requires only a tiny fraction of the ions available in this bi-compartmental system. Namely, only the mobile ions passing through the plasma membrane governs the membrane potential generation and its characteristics but the quantity of those ions has nothing to do with the membrane potential generation and its characteristics.

It follows from this statement that there is little change in the internal and external concentrations. 
It follows that the system can continuously diffuse several types of ions bidirectionally between the inside and outside by virtually causing no change in condition surrounding the cell.

All of these claims are totally disputable.

\section{Materials and Methods}

\subsection{Basic concepts of matter}

It is perhaps not useful to give a full account of the latest knowledge on matter. It is possible to be satisfied with the most common model that defines the smallest element of matter: the atom. This simplified (or even simplistic) model describes the atom as consisting of a nucleus and a cloud of electrons orbiting the nucleus [3]. The number of protons and neutrons that make up the nucleus and this cloud of electrons provides the chemical properties that we observe in chemical reactions and the products of these reactions. The composition of the nucleus and the number of electrons orbiting it also give us the electrical charge of that atom. If there are too many electrons in relation to the number of protons, the atom is of negative valence. If the number of protons exceeds the number of electrons, the atom is positive. And if the number of protons is equal to the number of electrons, the atom is neutral.

On a macroscopic scale, matter is electrically neutral. This is the perfectly accepted principle of electroneutrality [4]. It is also one of the fundamental principles of chemistry: Matter in a stable state must be electro-neutral. The number of positive ions or molecules $P_{i}^{+}(i=$ cationic species $)$ must be equal to the number of negative ions or molecules $N_{j}^{-}(j=$ anionic species) that make up this material. The electrical sum of these two numbers must be electro-neutral and therefore equal to zero.

$$
\sum_{i} z_{i} P_{i}^{+}+\sum_{j} z_{j} N_{j}^{-}=0 \quad z_{i}, z_{j}: \text { valency }
$$

Matter can also take on several states, and the biologically fundamental states are gas, liquid and solid. For example, water, which is very abundant on our planet, is solid in the form of snow, liquid in rivers, seas and oceans and vapour (gas) in the form of our clouds. The transition between these states is most often observed under the effect of temperature: most elements solidify when cold and become liquid when the temperature rises and end up as gas for some molecules when the temperature rises again.

\subsection{Components of a solution}

Chemistry also teaches us that some elements can change state through chemical reactions. These compounds are often present in a solvent in the dissolved form. Water is often considered a universal solvent and the large part of living things are accounted for by water and of course it serves as a solvent in the living things (see Fig.1). A solution is always composed of a solvent and the various products dissolved in it. 


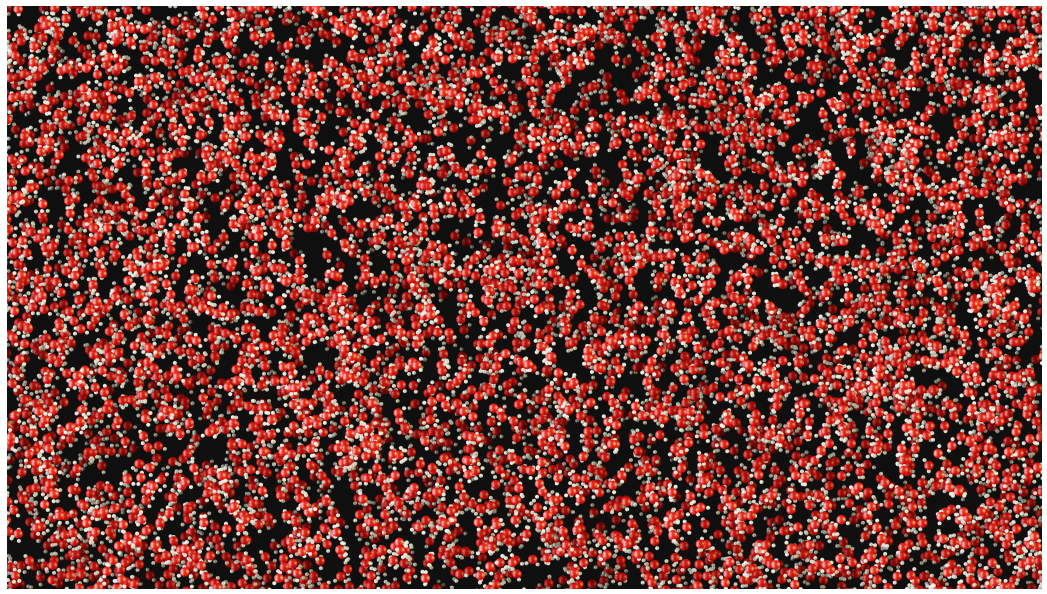

Figure 1. Artistic representation of the universal solvent, water. Only a few thousand molecules are illustrated.

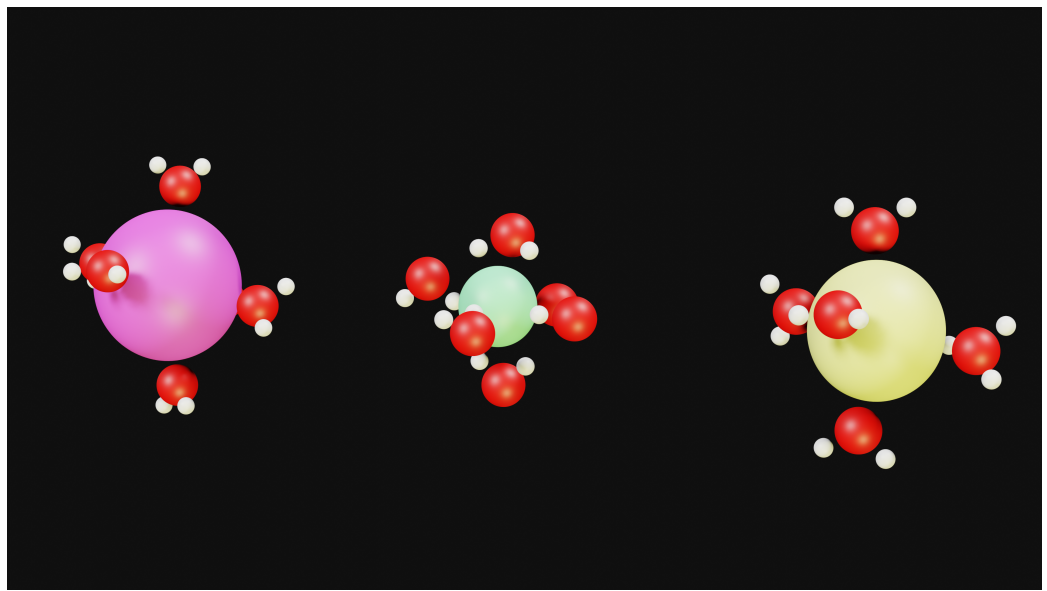

Figure 2. A $3 \mathrm{D}$ view of three hydrated ions. On the left, a potassium ion hydrated by 6 water molecules. The oxygen atoms are oriented towards the potassium. In the centre, a chlorine ion, the hydrogen atoms are oriented towards the anion. On the right, a sodium cation: the water molecules have the same orientation as for potassium. A single layer of hydration is shown in this image. Some molecules may have several layers of hydration which will slow down their mobility.

As mentioned above, on a macroscopic scale, matter is always electrically neutral. In the context of living beings, the solvent is always water, whose molecule is composed of two hydrogen atoms and one oxygen. It is then possible to write the chemical formula of water in two forms: the traditional one where valences are not taken into account and the current one which highlights the valence of the atoms and allows the distinction between cations and anions [5].

$$
\begin{array}{r}
\mathrm{H}_{2}+\frac{1}{2} \mathrm{O}_{2}=\mathrm{H}_{2} \mathrm{O} \\
2 \mathrm{H}^{+}+\mathrm{O}^{2-}=\mathrm{H}_{2} \mathrm{O} \\
(+1) \times 2+(-2) \times 1=0
\end{array}
$$

It is possible to check if the equation 1 is valid and to conclude that the water molecule is electrically neutral.But we must bear in mind that an electrically neutral water molecule can carry a non-zero electric polar property by retaining the polarisation caused by the spatial arrangement of the atoms.

When substances are dissolved in water, some remain neutral and others, such as salts, dissociate into their ionic components. The very common sodium chloride $\mathrm{NaCl}$ 
separates into the cation $\mathrm{Na}^{+}$and the anion $\mathrm{Cl}^{-}$. In addition to this dissociation, the ions are hydrated by water molecules (see Fig.2). A solution can be said to contain a certain amount of solvent and a certain amount of substance. It is usual to say that the more salts the solution contains, the more concentrated it is. A commonly used unit of measurement for concentration is the mole per litre $\left(\mathrm{mol} \cdot l^{-1}\right.$ or $\left.M\right)$.

$$
\begin{gathered}
c_{\mathrm{NaCl}}=[\mathrm{NaCl}]=\frac{n_{\mathrm{NaCl}}}{V} \\
N_{\mathrm{NaCl}}=n_{\mathrm{NaCl}} \cdot N_{A} \quad N_{A}: \text { Avogadro number }
\end{gathered}
$$

As the unit of concentration is related to a volume of one litre and the concentrations, in the case of a poly-electrolyte solution, add up, it is possible to say that the total concentration contained in the solution, although not very significant, is the sum of the different concentrations. For example, in biology, a solution can and often does contain several salts. If a solution is to contain both potassium and sodium chloride with a same concentration of $0.1 \mathrm{M}$, we mix a potassium chloride solution with a sodium chloride solution of equal concentration and volumes but the combined solution then contains two parts chlorine and its concentration is then $0.2 \mathrm{M}$.

$$
n_{\mathrm{KCl}}+n_{\mathrm{NaCl}} \underset{\text { dissociation }}{\longrightarrow} n_{\mathrm{K}}+n_{\mathrm{Na}}+2 n_{\mathrm{Cl}}
$$

Similarly, it is not possible to obtain a neutral mono-ionic solution. It is a simplification of language to speak of a potassium or sodium solution without taking into account its necessarily negative counterpart. In fact, we are not able to make a mono-ionic solution, which would be very unstable and dangerous because of the quantity of charges it would contain.

\subsection{Differences between molecular diffusion of neutral and ionic molecules.}

Diffusion is a phenomenon of matter transport, supposedly passive, linked to the thermal agitation of molecules. This transport of matter is irreversible and takes place between the most concentrated zone and the least concentrated. This tends to chemically homogenise the whole system. It is then also possible to say that simple diffusion has a direction and this introduces the notion of a vector in the mathematical and physical sense.

This notion of direction imposed by the factual and experimental conditions makes it easier to equate this movement of matter.

The units of diffusion are length squared divided by time, length ${ }^{2} /$ time, or $m^{2} s^{-1}$. And since we already know that a speed is the expression of a length divided by a time, we can also express diffusion as a length multiplied by a speed.

$$
D=\text { length } \times \text { length } / \text { time }=\text { length } \times \text { speed }=l \cdot v
$$

It is quite likely that the diffusion of neutral molecules is related to their density in the region under consideration and also to their thermal agitation, also called Brownian motion. The more important these two factors are, the higher the speed can be.

\subsubsection{Diffusion of neutral molecules}

Molecules with zero valence, known as neutrals, can follow the diffusion gradient and their direction is a function of the gradient vector. There is little or no chemical or electrical disturbance in the solution because the electrical charge in the solution is not changed. It is also possible to say that this direction depends on the shapes where these diffusion gradients are exercised. As the process involves a displacement of molecules in the space where this diffusion takes place, this process is neither instantaneous nor constant. While the calculation of the resulting concentrations is always easy, it may not represent a physical reality because diffusion does not lead to a homogeneous change in the zones. 
Similarly, measuring the concentration of a zone by sampling involves homogenising the sample and integrating the underlying physical phenomenon.

\subsubsection{Diffusion of charged molecules}

The diffusion of charged particles or ions can lead to the formation of an electrical gradient. As with neutral particles, diffusion is a function of the size of the charged ion or molecule. Diffusion is also a function of the electronegativity of the particle but also of its degree of solvation. Electronegativity is directly related to the valence of the ion and its size. The smaller the ion, the more electronegative it can be. Electronegativity is related to the surface area of the ion. It becomes clear that the valence of the ion can also increase this electronegativity: the higher the valence, the more electronegative the ion can be. Obviously, the champion ion is still the hydrogen ion. It has the highest mobility by size. Perhaps for this reason Walther Herman Nernst used its diffusive speed to demonstrate the diffusion potential in his experiments. As some ionic species diffuse faster than others, an electrical gradient will be created within the solution. This electrical gradient imposes the formation of an electric field which will limit and oppose this diffusion. This field is often called the electroneutrality field by chemists [5,6].

It is well known and proven that this phenomenon is limited. If the electric field is linked to the separation of charges then its growth will limit this same separation. Let's take the example where it is a positive ion that is more mobile than the negative ones.

- A positive ion leaves the neutral zone (see Fig.3).

- $\quad$ This creates two new zones; one negative and one positive.

- This creates an electrical gradient generating an electric field.

- With each new positive ion that leaves the zone, the electric field strengthens as the positive and negative zones strengthen.

- Each new positive ion that leaves the zone finds it increasingly difficult to leave the original zone.

- $\quad$ Equilibrium is reached when the electric field is strong enough to prevent mobility.

- Diffusion is stopped (see Fig.4).

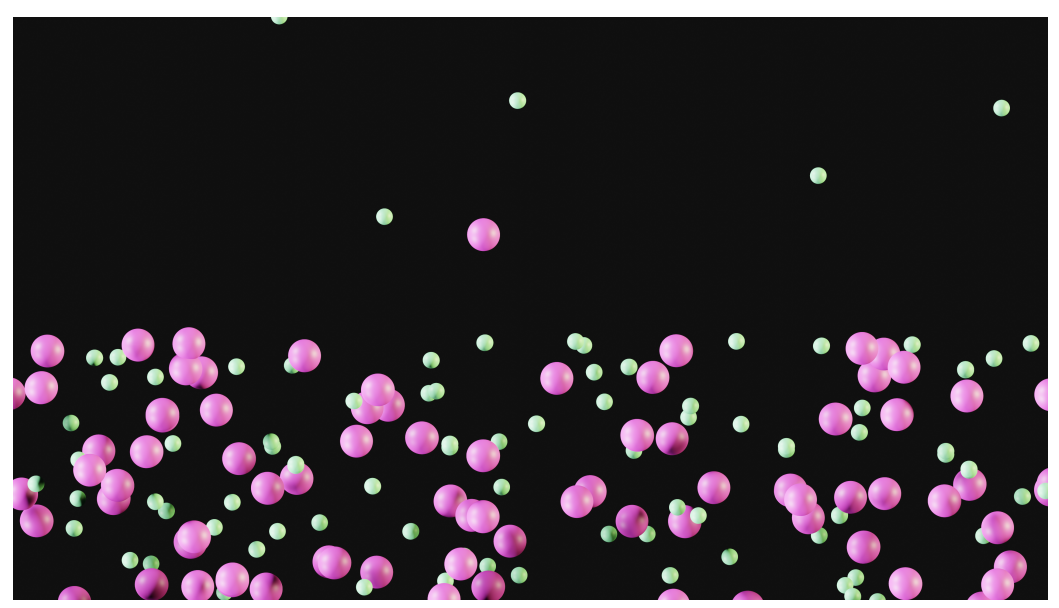

Figure 3. Initial condition of diffusion between two phases. The water molecules are not shown so as not to weigh down the figure or mask the ions. 


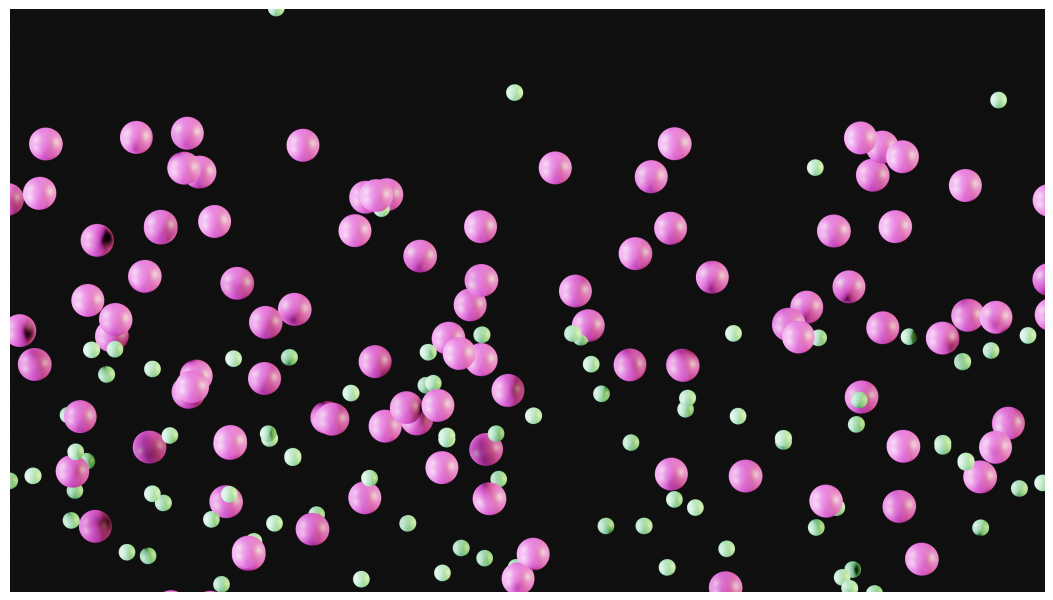

Figure 4. End of diffusion. Some of the positive ions diffused faster than the negative ones creating an electric field that stops the diffusion and the process. The water molecules are not shown so as not to weigh down the figure or mask the ions.

The presence of an electric field imposes a diffusion that remains stealthy in time. The system blocks itself $[7,8]$. Moreover, the electric field imposes a direction on all mobile charges. The positive ones tend to go towards the negative zone while the negative ones will try to go through the cathode zone. In addition, the movement of ions and charged particles is automatically accompanied by the movement of solvent molecules. The ions are hydrated and the hydration shells will move together with the ions.

\subsection{Nature and magnitude of the forces involved}

As mentioned earlier, the diffusion of neutral particles seems to be only related to the thermal agitation and density of the molecules. The smaller their size, the lower the velocity. This agitation leads to numerous collisions which are due to the erratic behaviour of the displacement. This displacement and the forces that drive it are therefore directly and intimately linked to the speed and mass of each molecule and their interactions with the solvent and other molecules that may be present in the solution. The mass of a hydrogen atom is of the order of $1.66 \cdot 10^{-27} \mathrm{~kg}$. It can be said that the resulting force of diffusion is the resultant, as it were, of the motion of the particle and its mass minus the resistances that the particle encounters within the solvent.

$$
\overrightarrow{F_{m}}=f(m \cdot v)-f(s o l)
$$

On the other hand, charged particles and ions add to this random thermal agitation a force directly proportional to their charge. The coordinated action of these forces is capable of creating an electric field that will be imposed on all charged particles. In addition, the hydrated layer that accompanies the ions also increases the range of the electrostatic action. In simple diffusion, it is the molecules, by their mass and speed, that bring about the change. In the case of charged particle diffusion, the electrical component, which is linked to the charge of the ion, must be added to this force.The elementary electric charge is $1.60 \cdot 10^{-19} \mathrm{C}$ (or $A s$ ). It is obvious that the electric force is always greater than the diffusion force. The diffusion force of an ion can be said to be the resultant of the motion caused by the electrical component of the particle and its mass reduced by the resistances that the particle encounters in the solvent, both mechanical and electrical.

$$
\begin{array}{r}
\overrightarrow{F_{\text {ion }}}=\overrightarrow{F_{m}}+f(z)-f\left(z_{\text {sol }}\right) \\
\overrightarrow{F_{\text {ion }}} \gg \overrightarrow{F_{m}}
\end{array}
$$




\section{Results}

The different phenomena we have described that are used in the MPT to explain the generation of the membrane potential are not compatible in the development of an ambitious and rigorous theory. Diffusion velocities are often compared but driven by forces of different magnitudes. This is not a logical or sound reasoning. A difference of a factor of $10^{+8}$ cannot be ignored or compared in a simple way.

Of course, it is not possible to make such a claim without further discussion by asking the right questions and, above all, by using rigorous logic. MPT has been built up bit by bit without questioning the previous elements of the theory. However, many elements already allowed us to raise doubts about the relevance of a logical sequence and a flawless functioning.

\section{Discussion}

\subsection{Logical origin of ions}

It can be stated without question that the origin of the ions present in the cells is always external to the cells. The ions can only come from the environment in which the cells are located. So we all agree that the external environment contains ions that are also contained within the cell. Of course, the concentrations of these different ions are not identical in the two compartments, internal and external.

If we believe the definition of the scientific model, it must be able to describe the observed facts and of course reproduce them as accurately as possible.

Let's take a fact that cannot be disputed: a cell grows and then divides into two daughter cells that grow and in turn divide... The cycle of life as we have observed it for as long as biology has existed. We can say that:

- Both mother $(A)$ and daughter (A.A and $A . B)$ cells have the same internal potassium concentration.

- The volume of the daughters $\left(V_{A . A}\right.$ and $\left.V_{A . B}\right)$ is approximately that of the mother $\left(V_{A}\right)$ divided by two.

- The same theory of generation and maintenance of membrane potential applies to the mother and daughter cells.

This can be translated into the following equations;

$$
\begin{array}{r}
\left|K^{+}\right|_{A}=\left|K^{+}\right|_{A . A}=\left|K^{+}\right|_{A . B} \\
V_{A . A}=V_{A . B}=\frac{V_{A}}{2}
\end{array}
$$

And with the equation 5 it is possible to deduce:

$$
n_{K_{A . A}}=n_{K_{A . B}}=\frac{n_{K_{A}}}{2}
$$

But as we know that the daughter cells will grow then their volume will grow too and the number of potassium ions must increase to keep the internal concentration stable (see Fig. 5) ;

$$
n_{K_{A . A}} \underset{\text { cell growth }}{\longrightarrow} n_{K_{A}}
$$

Throughout the growth of the cell, potassium enters the cell. However, for the duration of this growth, the cell is and remains fully functional. The MP is not affected in any way. It is not disputable that this potassium comes from the environment and therefore from outside the cell. 


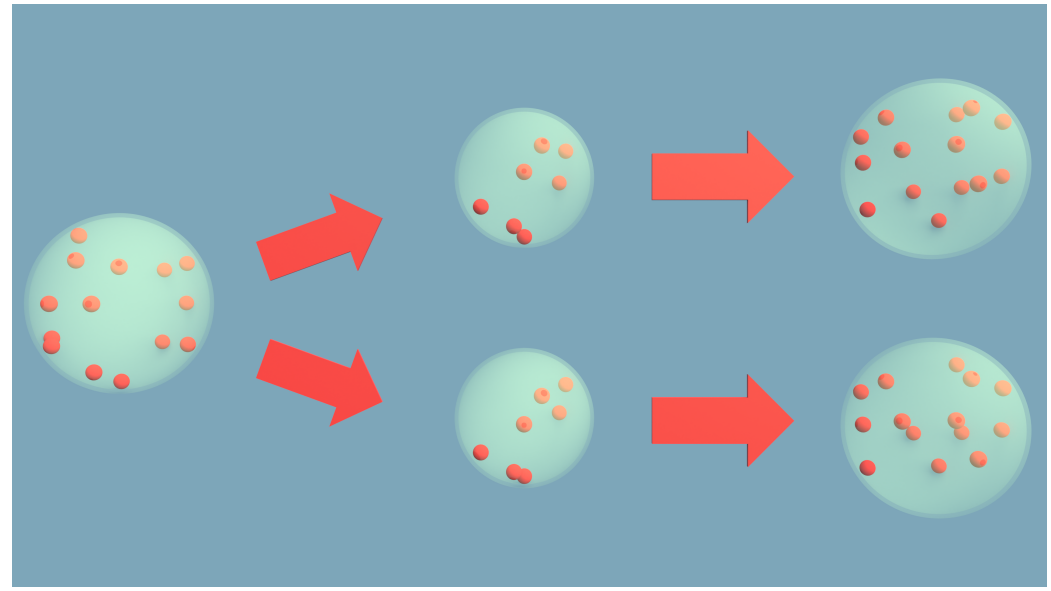

Figure 5. The cell growth cycle: A mother cell divides into two daughter cells that will grow in turn. The MP does not vary during and after division, nor does the internal potassium concentration. If the cell grows and the potassium concentration remains constant then it must be assumed that potassium must enter the cell.

It is therefore possible to affirm that during the entire growth of the cell and by extension of all multi-cellular living beings, potassium enters the cell in a massive way while allowing the continuation of essential functions and without any disturbance of the MP.

The MPT does not consider any kind of mechanism or hypothesis for this potassium influx. The $\mathrm{Na} / \mathrm{K}$ pump is even considered by its advocates to be minor in its involvement and less than $5 \%$. Is it possible to consider a hypothesis that potassium is continually leaving the cell when the facts and logic contradict it? Reason should rule out this mechanism.

\subsection{Utopian diffusion}

As we have just seen, the direction of the potassium gradient is contrary to this influx. But that's not all. Chemists and biologists agree on one point: If there is a concentration gradient, diffusion takes place from the most concentrated area to the least concentrated AND this diffusion stops when the concentrations balance out. Chemists are even stricter because in the case of ion diffusion, they observe that this diffusion is limited and slows down to a halt by the presence of the electric field of electroneutrality. Even if the potassium had the right direction and as it comes from the external medium, the theory imposes a limit which must be equal to the value of the external concentration. This limit is largely exceeded in proportions that leave no doubt: 30 times!

Figure 6 shows the initial situation as our devices appear to measure it, but we take care to separate the various components to achieve this. It is not certain that the location is accurate and consistent. It is perfectly accepted that the ions bind to the proteins and are not mobile at all. 


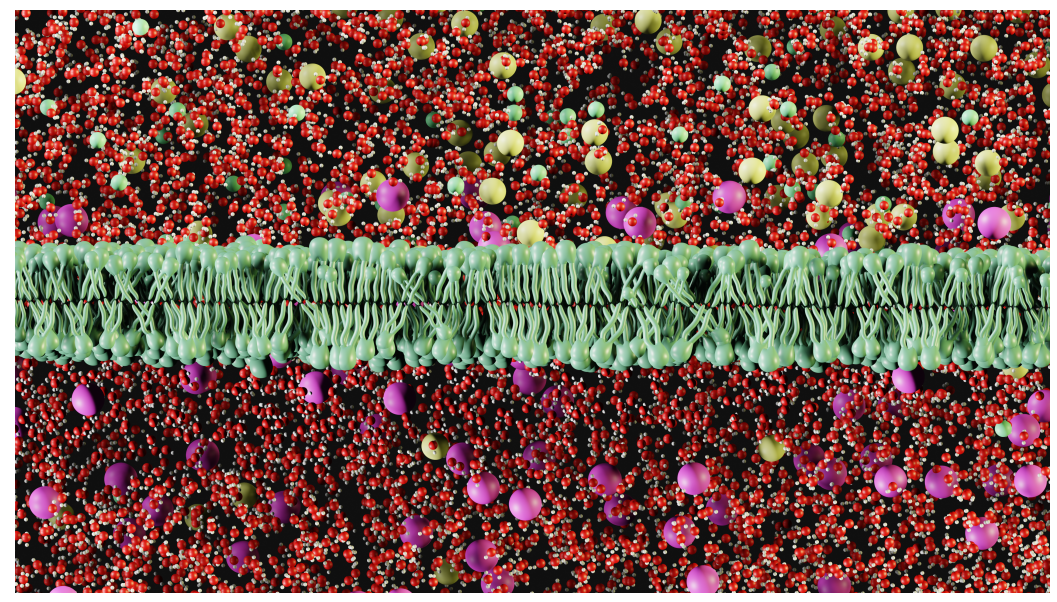

Figure 6. Initial conditions of the membrane potential (or resting potential). Almost all mobile ions are shown $(\mathrm{Na}, \mathrm{K}, \mathrm{Cl})$. The water molecules that form the solvent are also shown. This picture shows the complexity and difficulty that diffusion of several types of ions can take place independently.

Figure 7 shows the same starting situation. This is the one often shown in biology textbooks. The situation has been simplified by omitting an element that is essential for many biological reactions and processes: water. This simplification is often necessary to highlight a key point of the theory, but this reductionism can also make us forget the complexity of the situation and the importance of an actor who is reputed to be neutral but who is in fact the opposite.

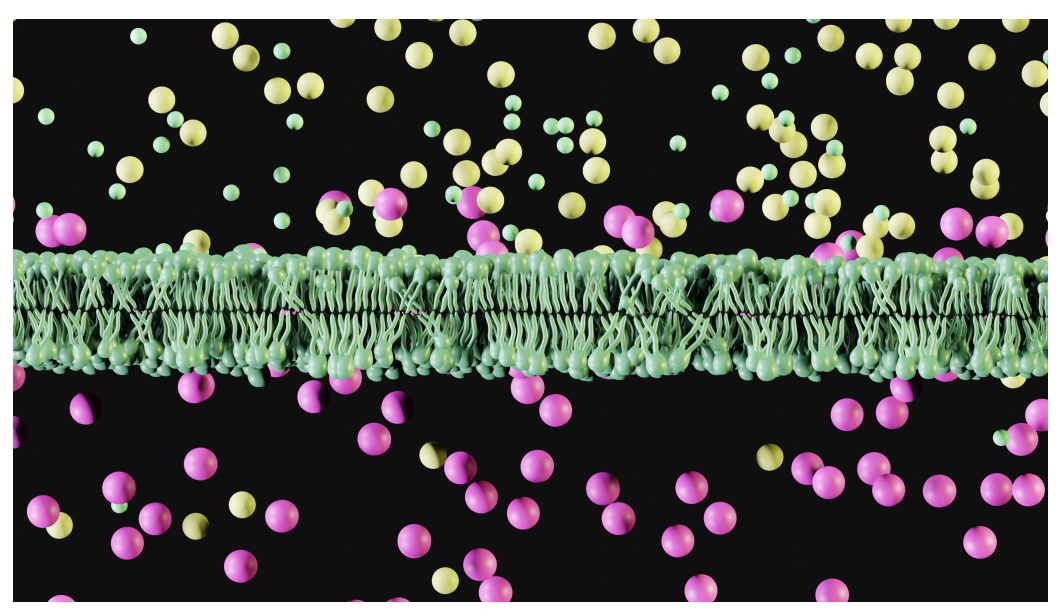

Figure 7. Initial conditions of the membrane potential (or resting potential). Almost all mobile ions are shown $(\mathrm{Na}, \mathrm{K}, \mathrm{Cl})$. The solvent is no longer shown. The excess of potassium outside the cell (top) is clearly visible. We can also see that the sodium is more concentrated outside the cell.

This concentration coefficient is out of reach of a possible action of the $\mathrm{Na} / \mathrm{K}$ pump as we have already said. As has been pointed out, the ions are hydrated and carry water molecules in their movements that escape any hypothesis of the MPT. These ionic movements can only disrupt the other diffusions that are imagined in the theory. How is sodium able to enter the cell when the electric field prevents it? The simplest answer is that it does not enter because it cannot, unlike potassium whose entry is favoured or facilitated.

How can sodium enter without affecting the potassium ions that would be on the surface of the cell? Aren't the sodium ions repelled? Doesn't the movement of sodium cause a movement of chlorine as observed in chemistry? The theory developed by Hodgkin and Huxley and by Katz does not attempt to answer these kinds of trivial but essential questions. There are enough alternative theories, even validated by experience $[4,9,10]$, which demonstrate another truth, simpler and more precise, and which, moreover, allows 
a dynamism which cannot exist due to the presence of the electric field considered as uniform and constant.

In any case, the diffusion theory is not respected in the MPT in any way. Indeed, it is claimed that diffusion takes place from the more concentrated $c_{h i g h}$ to the less concentrated $c_{\text {low }}$ and this in a passive way. It is expected that the intermediate centration $c_{\text {mid }}$ should be between the two values mentioned above. However, it is stated that the ions are dehydrated before entering the ion channels. A dehydration of ions is an increase in concentration in massive proportions.

$$
c_{\text {mid }} \gg c_{\text {high }}>c_{\text {low }}
$$

The process described has nothing to do with diffusion and cannot use its mathematical laws. If diffusion were the engine of MPT then ions would diffuse out of the ion channels both into and out of the cell.

\subsection{The concept of interdependency}

As it is not possible, at a given time $t$, to separate the solution into its original components, it is possible that all the ions present may interact with a specific species that the observer would like to isolate in his hypotheses.

At any given moment the membrane would be the seat of outgoing and incoming ionic flows that we know collide with other molecules and ions that are present, by definition. Shouldn't we assume some kind of interaction between these different flows? Should we not assume that these flows are hindered or even prevented by the presence of flows in the opposite direction? [11,12]

However, these contrary movements are not considered in the Goldmann, Hodgkin and Katz equation. Why does the latter not take into account the direction of the electric field? This is a question that few people ask, but which calls into question its validity!

The MPT does not embrace this kind of blatant contradiction. The ions are independent because the water molecules separate them although they create an electric field and although the water molecule also has an ionic/electrostatic behaviour due to its assymetrical form.

These claims are clearly contradicted by chemistry and industry.

It should not be forgotten that biology, even if it has industrial and medical spin-offs, is largely competed by manufacturing processes that are used worldwide and based on proven and logical theories.

\section{Conclusions}

Diffusion is considered to be the source of the membrane potential. We have demonstrated by example and also mathematically that potassium enters the cell during its growth contradicting the assumptions of the MPT. We also explored the sciences (chemistry and electrochemistry) that are also used by biology and biophysics and showed that some of the phenomena described cannot exist. We have also shown that the diffusion described is a phenomenon that does not meet any of the criteria described in chemistry. It is time to change the paradigm and find a theory that respects the facts and that can finally have a logical sequence in its dynamics. The potassium does not seem to leave the cell but on the contrary seems to be always accepted by negative components which remain numerous inside the cell and also in the membrane. These proteins are of course the ion channels and the cytoskeleton. However, their function needs to be reviewed as it seems to be contradicted by the facts.

Author Contributions: Conceptualization, B.D. and H.T. and V.M.; investigation, B.D.; writingoriginal draft preparation, B.D. and H.T. and V.M; writing —review and editing, B.D. and H.T. and V.M; visualization, B.D.; All authors have read and agreed to the published version of the manuscript.

Conflicts of Interest: The authors declare no conflict of interest. 


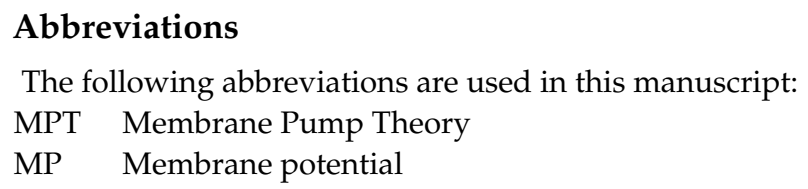

\section{References}

1. Bernstein, J. Untersuchungen zur Thermodynamik der bioelektrischen Ströme. Pflüger Archiv für die Gesammte Physiologie des Menschen und der Thiere 1902, 92, 521-562. doi:10.1007/bf01790181.

2. Goldman, D.E. POTENTIAL, IMPEDANCE, AND RECTIFICATION IN MEMBRANES. Journal of General Physiology 1943, 27, 37-60. doi:10.1085/jgp.27.1.37.

3. Barrow, G.M. Physical Chemistry; McGraw-Hill Science, Engineering \& Mathematics, 1996.

4. Ling, G. Debunking the alleged resurrection of the sodium pump hypothesis. Physiological chemistry and physics and medical NMR, 29.

5. Bockris, J.O.; Reddy, A.K.; Gamboa-Adelco, M.E. Modern Electrochemistry 1, 2A, and 2B; Springer, 2006.

6. Delalande, B.; Tamagawa, H.; Mulembo, T.; Matveev, V. Inevitable Variance of Electric Field of Plasma Membrane.

7. Delalande, B.; Tamagawa, H.; Matveev, V. The Proof by 1 to n. doi:10.20944/preprints202106.0356.v1.

8. Delalande, B.; Tamagawa, H.; Matveev, V. From Nernst to Bernstein and Beyond. OALib 2020, 7, 1-18. doi:10.4236/oalib.1107047.

9. Ling, G.N. A Revolution in the Physiology of the Living Cell; 1992.

10. Ling, G.N. Life at the Cell and Below-Cell Level: The Hidden History of a Fundamental Revolution in Biology; Pacific Press, Incorporated, 2001.

11. Hodgkin, A.L. Evidence for electrical transmission in nerve 1. The Journal of Physiology 1937, 90, 183-210. doi:10.1113/jphysiol.1937.sp003507.

12. Hodgkin, A.L. Evidence for electrical transmission in nerve 2. The Journal of Physiology 1937, 90, 211-232. doi:10.1113/jphysiol.1937.sp003508. 
